\title{
IMAGERIE ULTRASONORE QUANTITATIVE
}

\author{
P. GRASSIN et B. DUCHENE \\ Equipe Electromagnétisme, Laboratoire des Signaux et Systèmes, CNRS ESE, Plateau de Moulon, \\ F-91192 Gif-sur-Yvette cedex, France
}

\begin{abstract}
We investigate herein transmission mode ultrasonic imaging of 3-D fluid media from single-frequency multiview synthetic data. The technique used is based upon a Newton-Kantorovitch algorithm, which yields an iterative solution of the non-linear ill-posed inverse scattering problem, through a linearization and a Tikhonov regularization procedure. We lay emphasis on the robustness of the algorithm.
\end{abstract}

\section{INTRODUCTION}

Nous considérons ici l'imagerie ultrasonore d'objets tridimensionnels limités (3-D), à partir de données simulées, collectées en transmission à une seule fréquence et pour différentes illuminations. Nous nous plaçons dans le domaine de résonance où les inhomogénéités des milieux étudiés ont des dimensions de l'ordre de grandeur de la longueur d'onde interrogatrice, et dans lesquels, par conséquent, les phénomènes de diffraction sont importants. Nous visons à obtenir une image d'un objet à partir de la mesure du champ résultant de son interaction avec une onde incidente connue, et au travers d'une modélisation de cette interaction tenant compte des phénomènes de diffraction. Une telle modélisation conduit à une représentation intégrale des champs que nous présentons dans la première partie de ce travail, consacrée à l'étude du problème direct, où l'objet étant connu, on s'intéresse au calcul du champ diffracté. Cette étape est indispensable pour l'obtention des données synthétiques du problème inverse. Le "problème inverse de diffraction" est fortement non-linéaire et mal posé. Son caractère non-linéaire est abordé à l'aide d'une technique dite de Newton-Kantorovitch, qui permet de construire une solution de façon itérative [1, 2, 3], en résolvant alternativement le problème direct et le problème inverse localement linéarisé. Son caractère mal-posé est, quant à lui, abordé à l'aide d'une technique de régularisation standard de Tikhonov. [4]. Cette dernière met en oeuvre un paramètre de régularisation dont la maîtrise est délicate. Dans le cas de données bruitées, une valeur optimale de ce paramètre peut être déterminée à l'aide de la méthode dite de validation croisée généralisée $[5,6]$, qui permet une convergence rapide du processus vers une solution stable.

\section{LE PROBLEME DIRECT}

Considérons un objet 3-D fluide $\Omega$ caractérisé par une vitesse des ondes de compression $\mathrm{C}_{\Omega}(\mathrm{x}, \mathrm{y}, \mathrm{z})$ et une atténuation $\sigma_{\Omega}(\mathrm{x}, \mathrm{y}, \mathrm{z})$ dépendant des coordonnées d'espace. Cet objet est plongé dans un milieu fluide homogène sans pertes $D_{0}$ (de vitesse $C_{0}$ constante) et est illuminé par une onde incidente plane $P_{0}$ en régime harmonique (pulsation $=\omega$, dépendance temporelle $=\mathrm{e}^{-\mathrm{j} \omega \mathrm{t}}$ ) dont la direction de propagation $\overrightarrow{\mathrm{k}}_{0}$, située dans le plan $x-y$, fait un angle $\theta$ avec l'axe des $x$. Les constantes de propagation dans l'objet et dans le milieu hôte sont respectivement désignées par $\mathrm{k}_{\Omega}(\mathrm{x}, \mathrm{y}, \mathrm{z})\left(=\omega / \mathrm{C}_{\Omega}+\mathrm{i} \sigma_{\Omega}\right)$ et $\mathrm{k}_{0}\left(=\omega / \mathrm{C}_{0}\right)$, les fluctuations de densité sont négligées. Le champ de pression scalaire $P(M)$ observé en tout point $\mathbf{M}=(\mathrm{x}, \mathrm{y}, \mathrm{z})$ est donné par la représentation intégrale suivante : 


$$
P(M)=P_{0}(M)+\int_{\Omega} \chi(M) P(M) G\left(M, M^{\prime}\right) d M^{\prime}, \chi(M)=k_{\Omega}^{2}(M)-k_{0}^{2}
$$

où $\mathrm{P}_{0}$ représente le champ incident, c'est-à-dire le champ en l'absence d'objet, $\chi$ représente le contraste, et $G$ la fonction de Green de l'espace libre 3-D.

Nous cherchons ici à calculer le champ diffracté sur un plan de mesure, l'objet $\Omega$ et le champ incident $P_{0}$ étant connus. Celui-ci peut être obtenu à partir de l'équation (1) en appliquant la méthode des moments. $\Omega$ est alors discrétisé en $\mathrm{N}$ cellules cubiques élémentaires $\Delta_{\mathrm{i}}$ de même taille, et de côté $\delta \Delta$ de longueur petite devant la longueur d'onde $\lambda_{0}\left(\delta \Delta\right.$ est de l'ordre de $\left.\lambda_{0} / 7\right)$. Dans chaque cellule, le contraste et la pression sont considérés comme constants. On obtient alors $N$ équations linéaires où les inconnues sont les valeurs du champ au centre $M_{i}$ de chacune des cellules élémentaires $\Delta_{i}$ :

$$
P\left(M_{i}\right)=P_{0}\left(M_{i}\right)+\sum_{j=1}^{N} \chi\left(M_{j}\right) P\left(M_{j}\right) G_{i}\left(M_{i}, M_{j}\right), G_{i}\left(M_{i}, M_{j}\right)=\int_{\Delta_{j}} G\left(M_{i}, M^{\prime}\right) d M^{\prime}
$$

Le système linéaire (2) est résolu à l'aide d'une technique de gradient-conjugué ou d'un développement du champ en série de Neumann (dans le cas de faibles contrastes). Une fois le système (2) résolu, le champ peut aisément être calculé en tout point $\mathrm{M}_{\mathrm{r}}$ du plan de mesure à l'aide de l'analogue discret de (1), le champ $P\left(M_{i}\right)$ à l'intérieur de l'objet étant connu.

\section{LE PROBLEME INVERSE}

Nous nous interessons maintenant au problème inverse, où, à partir de mesures en transmission du champ $P\left(M_{r}\right)$ sur un plan perpendiculaire $a ~_{0}$, il s'agit de reconstruire une image de l'objet, c'est-à-dire une cartographie des paramètres de l'objet. Plaçons nous dans un espace de Hilbert approprie, muni d'un produit scalaire et d'une norme associée notée $\mid I$. II, et réécrivons l'équation (1) en termes d'opérateurs et de fonctions :

$$
\Psi=A \chi
$$

Nous cherchons à obtenir le contraste $\chi$ à partir d'une inversion de l'équation (3), le champ diffracté $\Psi=\mathrm{P}-\mathrm{P}_{0}$ étant connu. Ce problème est fortement non-linéaire et mal-posé, la première étape consiste donc à le linéariser. En négligeant les quantités de second ordre, les variations $\delta \Psi$ du champ diffracté $\Psi$, induites par de petites variations $\delta \chi$ du contraste $\chi$, sont alors linéairement reliées à $\delta \chi$ :

$$
\delta \Psi=D \delta \chi \quad \text { où } \quad D=G_{r}\left(I-\chi G_{i}\right)^{-1} P
$$

$\mathrm{G}_{\mathrm{r}}$ étant défini de façon semblable à $\mathrm{G}_{\mathrm{i}}(2), \mathrm{M}_{\mathrm{r}}$ remplaçant $\mathrm{M}_{\mathrm{i}}$, et $\mathrm{P}$ représentant le champ à l'intérieur de l'objet. Pour remédier au caractère mal-posé du problème, une procédure de régularisation standard de Tikhonov est appliquée. II s'agit alors de trouver une solution $\delta \chi$ telle que :

$$
\|D \delta \chi-\delta \Psi\|^{2}+\alpha\|\delta \chi\|^{2}=\min
$$

où $\alpha$ est un paramètre de régularisation. Une telle solution est donnée par :

$$
\delta \chi=\left(D^{*} D+\alpha I\right)^{-1} D^{*} \delta \Psi
$$


où I représente l'identité et $\mathrm{D}^{*}$ l'adjoint de $\mathrm{D}$. La solution $\chi$ est alors construite itérativement :

1) une valeur initiale $\chi_{0}$ du contraste $\chi_{m}$ est choisie : $\chi_{m}=\chi_{0}$

2) le problème direct (2) est alors résolu avec ce $\chi_{m}$, ce qui nous donne le champ diffracté $\Psi_{m}$

3) on en déduit l'écart $\delta \Psi_{m}=\Psi_{m}-\Psi$ entre le champ $\Psi_{m}$ ainsi caiculé et les données $\Psi$

4) on cherche alors la solution $\delta \chi_{\mathrm{m}}$ de (5) qui correspond à cet écart $\delta \Psi_{\mathrm{m}}$, on obtient ainsi la nouvelle estimée $\chi_{\mathrm{m}+1}=\chi_{\mathrm{m}}+\delta \chi_{\mathrm{m}}$, puis on retourne à l'étape 2) avec $\mathrm{m}=\mathrm{m}+\mathrm{l}$.

La procédure est arrêtée quand l'écart $\delta \Psi_{\mathrm{m}}$ entre le champ calculé et les données est jugé "suffisamment" petit. Cet écart est mesuré par la quantité ERR $(\Psi)=\left(\left\|\delta \Psi_{\mathrm{m}}||^{2} /\right\| \Psi \|\left.\right|^{2}\right)^{1 / 2}$.

La prise en compte de plusieurs vues (celles-ci étant obtenues en mesurant le champ diffracté $\Psi$ pour différents angles $\theta$ ) permet une convergence plus rapide du processus, quoique le volume de calcul global soit beaucoup plus important; $\delta \Psi$ contient dans ce cas les données correspondant aux différentes vues, qui sont alors traitées simultanément, $\mathrm{D}$ étant modifié en conséquence.

Notons que $\mathrm{D}$ et $\alpha$ sont révvalués à chaque itération $\mathrm{m}$. Lorsque les données ne sont pas bruitées, la valeur de $\alpha$ peut être déterminée intuitivement en considérant que la régularisation doit être forte au début de la procédure, lorsque nous sommes loin de la solution, et doit progressivement décroître au fur et à mesure que nous nous rapprochons de cette dernière, de façon à éviter une perte de résolution. Un choix $\alpha \propto \operatorname{ERR}^{2}(\Psi)$ permettra alors d'obtenir rapidement une solution satisfaisante. Cependant, il n'en va plus de même lorsque les données sont bruitées (Cf. Figs. 1 et 2). Dans ce cas, une valeur optimale du paramètre de régularisation peut être déterminée à l'aide d'un algorithme de validation croisée généralisée. Cette méthode consiste à omettre une observation (une valeur du champ diffracté $\Psi$ ) pour le calcul de la solution régularisée (6), puis à prédire cette observation à l'aide de la solution calculée à partir des autres observations. La valeur optimale de $\alpha$ est alors celle qui assure la meilleure prédiction, en moyenne, sur toutes les omissions possibles. L'intérêt de cette méthode est qu'elle ne nécessite aucune connaissance a priori sur le bruit. Elle permet, d'autre part, de converger rapidement vers une solution stable satisfaisante même avec des données fortement bruitées.

Résultats numériques : L'objet étudié est un cube homogène sans atténuation, de vitesse $C_{\Omega}=1560 \mathrm{~m} / \mathrm{s}$, de côté égal à $\lambda_{0}$ et discrètisé en $5 \times 5 \times 5$ cellules cubiques. Il est insoné à une fréquence de $2 \mathrm{MHz}$ et plongé dans l'eau $\left(C_{0}=1470 \mathrm{~m} / \mathrm{s}\right.$, longueur d'onde $\left.\lambda_{0}=.735 \mathrm{~mm}\right)$. Le champ diffracté est calculé sur un plan situé à une distance de $3 \lambda_{0}$ du centre de l'objet, en $16 \times 16$ points (pas d'échantillonnage $=\lambda_{0} / 2$ ). Ces données sont alors bruitées avec un rapport signal à bruit de $20 \mathrm{~dB}$. L'algorithme de Newton Kantorovitch est appliqué sans information a priori sur la solution, toutes les cellules de l'objet étant initialement considérées comme constituées d'eau. La reconstruction est effectuée à partir de 8 vues; l'écart

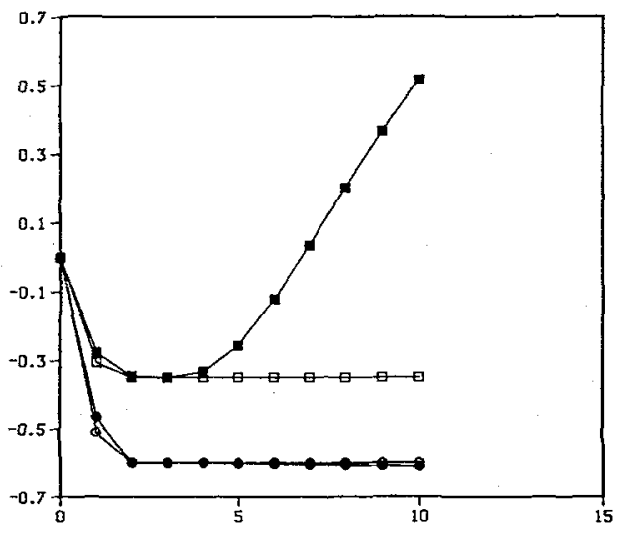
angulaire entre vues est $\Delta \theta=45^{\circ}$.

Figure 1: l'erreur normalisée sur le contraste $\chi$ $\left(\log _{10} E R R(\chi)=\right.$ carrés $)$ et sur le champ $\Psi$ $\left(\log _{10} E R R(\Psi)=\right.$ ronds) en fonction du nombre d'itérations au cours du processus appliqué avec deux choix différents du paramètre de régularisation:

1) une évolution de $\alpha$ telle que $\alpha \propto E R R^{2}(\Psi)$ (symboles noirs)

2) une évolution de $\alpha$ déterminée par validation croisée généralisée (symboles blancs) 
La figure 1 décrit l'évolution de $\operatorname{ERR}(\Psi)$ et $\operatorname{ERR}(\chi)$ au cours du processus. $\operatorname{ERR}(\chi)$ est définie de façon similaire à $\operatorname{ERR}(\Psi), \chi$ remplaçant $\Psi$, et décrit l'erreur sur la solution. Avec un choix de l'évolution du paramètre de régularisation en $\alpha \propto \operatorname{ERR}^{2}(\Psi)$, on observe que l'erreur sur la solution $\operatorname{ERR}(\chi)$ diminue et atteint sa valeur minimale à la $3^{\text {ième }}$ itération puis augmente après la $4^{\text {ième }}$ itération. La solution est donc instable, et l'évolution de ERR( $\Psi)$, la seule quantité accessible en pratique, ne présente pas de traits caractéristiques permettant de repérer l'itération pour laquelle $\operatorname{ERR}(\chi)$ est minimale, c'est-à-dire pour laquelle l'image obtenue est la meilleure. La figure 2 montre, pour sa part, des coupes longitudinales (x-y)

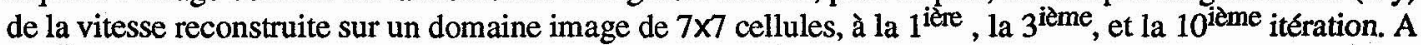
la $3^{\text {ième }}$ itération, les vitesses obtenues coïncident avec les valeurs théoriques avec une erreur de $\pm 40 \mathrm{~m} / \mathrm{s}$; par contre, à la $10^{\text {ième }}$ itération les vitesses reconstruites n'ont plus rien à voir avec les vitesses recherchées. Lorsque la méthode de validation croisée généralisée est appliquée pour déterminer la valeur optimale du paramètre de régularisation, nous observons que $\operatorname{ERR}(\chi)$ et $\operatorname{ERR}(\Psi)$ diminuent au cours des itérations et finissent par devenir constantes (Cf. Fig.1). La solution est stable et l'image "définitive" de l'objet, sur laquelle nous observons les vitesses théoriques avec une erreur de $\pm 40 \mathrm{~m} / \mathrm{s}$, est obtenue dès la $1^{\text {ière }}$ itération (Cf. Fig.2), l'image restant quasiment inchangée au cours du processus.

évolution du paramètre de régularisation $\propto \operatorname{ERR}^{2}(\psi)$
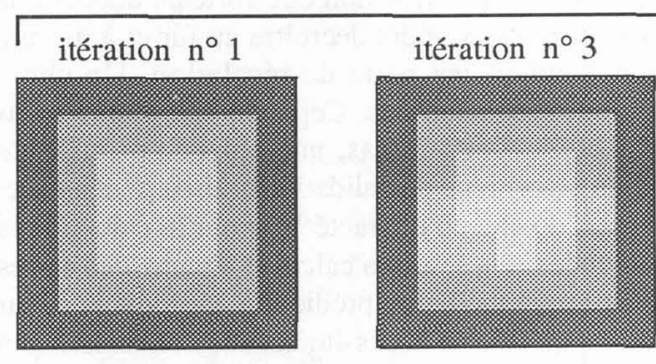

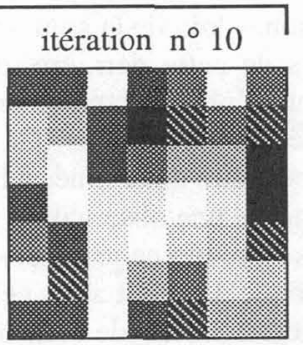

validation croisée itération $\mathrm{n}^{\circ} 1$

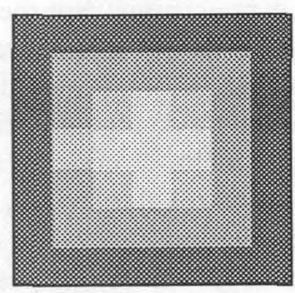

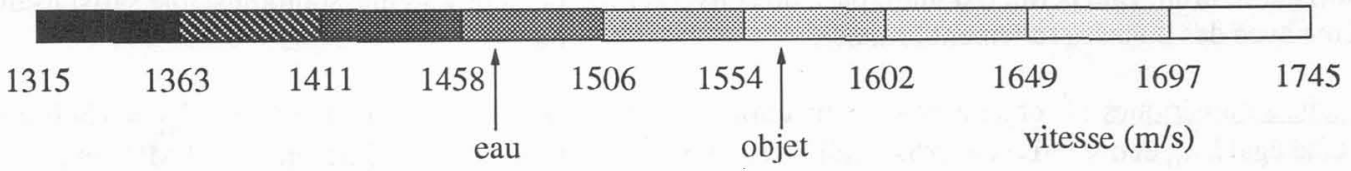

Figure 2 : image d'un cube de côté $\lambda_{0}$ reconstruite à partir de 8 vues. Coupe $(x-y)$ de la vitesse reconstruite au cours du processus pour deux choix différents du paramètre de régularisation $\alpha$.

\section{REFERENCES}

[1] A. Roger, "Newton-Kantorovitch algorithm applied to an electromagnetic inverse problem", IEEE Trans. Antennas Propagat., 29, pp. 232-238, 1981.

[2] J.P. Hugonin et al., "Quantitative reconstruction of complex permittivity distributions by means of microwave tomography", in Inverse Methods in Action, P.C. Sabatier Ed., Springer-Verlag, Berlin, pp. 302-310, 1990.

[3] P. Grassin et B. Duchêne, "Imagerie ultrasonore qualitative et quantitative en milieux 3-D", Proc. Rencontre Acoustique Sous-Marine et Ultrasons, ISSN 0750-7356, pp. 359-371, Marseille, 1991.

[4] A.N. Tikhonov et V.Y. Arsanine, in Méthodes de Résolution de Problèmes Mal-posés, Mir, Moscou, 1976.

[5] G.H. Golub, M. Heath, G. Wahba, "Generalized cross-validation as a method for choosing a good ridge parameter", Technometrics $\odot, 21$, no. 2, pp. 215-223, Mai 1979.

[6] D. Girard, "Practical optimal regularization of large linear systems", Mathematical Modelling and Numerical Analysis, $20, \mathrm{n}^{\circ} .1$, pp. 75-87, 1986. 